\title{
Possibility of an Electrophysiological Experimental model using Brainstem Evoked Potentials with multi-channel dural electrodes array in rats
}

\author{
Yuki Tadokoro \\ Ichikawa High School, 2-38-1 Motokitakata, Ichikawa, Chiba, 272-0816 Japan \\ TEL: +81-47-339-2681 FAX: +81-47-337-6288
}

Mitsuaki Ohtsuka

Graduate School of Biomedical Engineering, Tokai University, 317 Nishino, Numazu, Shizuoka, 410-0395 Japan

TEL: +81-55-968-1111 FAX: +81-55-968-1156 e-mail: mitsuaki052000@yahoo.co.jp

\section{Tatsuhiro Kimura}

Department of Information and Communication Technology, Tokai University, 317 Nishino, Numazu, Shizuoka, 410-0395 Japan

TEL:+81-55-968-11111 FAX:+81-55-968-1156 e-mail: kimuta@wing.ncc.u-tokai.ac.jp

Katsuro Okamoto, Kiyoyuki Yamazaki and Hiroyuki Tadokoro

Department of Biomedical Engineering, Tokai University, 317 Nishino, Numazu, Shizuoka, 410-0395 Japan

TEL:+81-55-968-1111 FAX: +81-55-968-1156 e-mail:tadokoro@tokai-u.jp

( Received 8, February 2010 Accepted 1, September 2010)

The rat is one of the most popular experimental animals in medical fields. However, the rat is not so often used in complicated neurological studies because of its size of the brain. To investigate the localization of waveform of auditory brainstem responses (ABRs), two rats were examined. Twelve or 13 platinum-rhodium coated electrodes were implanted on the dura mater through the cranial bone. The waveform of ABRs with 6 peaks was clearly recorded. These peaks were comparable with those of the human. However, the latency of these peaks was slightly shorter compared with that of the human. These 6 peaks were clearly identified in the waveform measured on the occipital area. On the other hand, these peaks recorded in frontal region were fused together. From these results, electrodes should be placed on the occipital area of the cranial bone to identify these peaks originated from brainstem by the auditory stimulation. Therefore, it is expected that latency changes can be evaluated clearly. This measurement system has a new possibility to analyze the details of various kinds of evoked potentials waveforms.

Keywords: Brainstem, Auditory evoked potentials, Rat, Chronic animal model

\section{INTRODUCTION}

In the past, rodents were classified into poorly intelligent mammals. Therefore, they have not been

SAS Award was given to this paper presented as Poster

Presentation at the 2009 SAS Intelligent Symposium. regarded as suitable for brain researches. The anatomical and physiological research of the rodent's brain made rapid progress in the 90s. As a result, it became clear that there was a little difference between rodents and higher organism like monkeys. Recently, mice and rats are widely used in cerebral 
neuronal physiological researches ${ }^{1}$.

Auditory brainstem responses (ABRs) of rats were commonly used to test the auditory nerve function ${ }^{2-3}$. Until now, the quality of ABRs waveforms was not evaluated well. We developed new ABRs measurement system using rats with dural electrodes $^{4-5}$. Moreover, large differences of ABRs wave-shape were observed between adjacent electrodes, which are several millimeters away.

In this study, the experimental model in rats with implanted multi electrode array was developed by precise surgical operation. In order to investigate the feasibility of this experimental system, the ABRs were recorded and analyzed. The relationships between spatial distributed characteristics of ABRs and neural processing of auditory stimuli were also analyzed.

\section{METHODS}

The following experimental protocol was approved by animal ethics committee in Tokai University.

Two 8-week-old Wister rats (CLEA Japan, Inc.) were used in this experiment. After anesthetized with pentobarbital sodium $(40 \mathrm{mg} / \mathrm{kg}$, ip), the hair of head was clipped. After this point, all surgical procedures were performed under aseptic condition.

The skin of head was incised longitudinally, and fascia temporals were removed from the cranial bone. According to the "The Rat Brain in stereotaxic coordinates”, 12 holes in rat A and 13 holes in rat B were cut with a hand drill ${ }^{6}$ (Fig.1). Thin silver coated cupper wires were fixed on the skull with platinum rhodium coated screw (Fig. 2). In rat A, bare wires were used, on the other hand, Teflon coated wires were selected in rat B (Fig. 3). In rat A, to measure lateral side electrical activities, probes were placed on the temporal lobe of the brain. In rat $\mathrm{B}$, to examine laterality, the probes were placed both side of the brain. The reference electrode was sutured to the latissimus dorsi muscle. The grounding electrode was placed forehead of the brain as the same manner of probes. After these procedures, screw and wires were covered with epoxy resin to fix and cover the cranial bone (Fig. 3). No antibiotics were used.
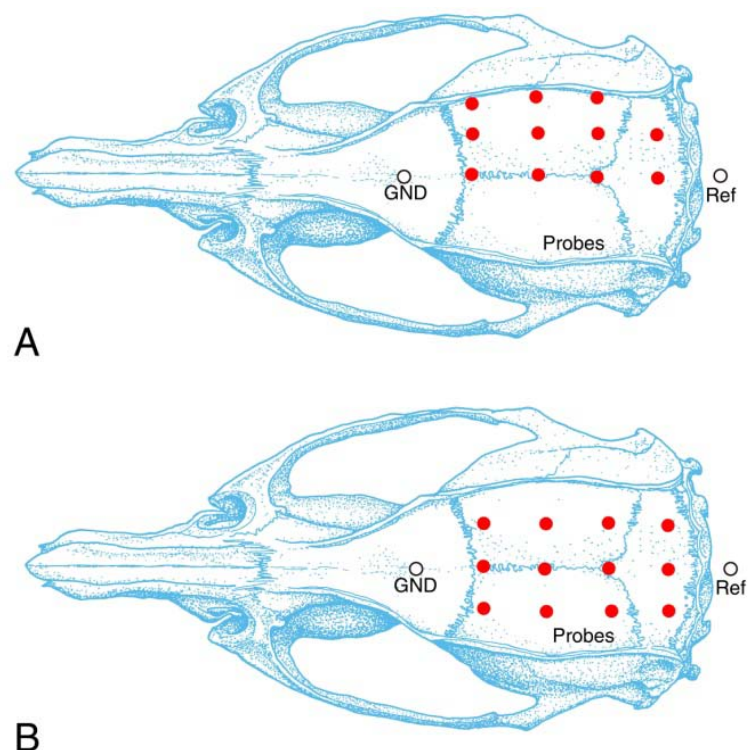

Fig. 1. The alignment of electrode in rat A and B.

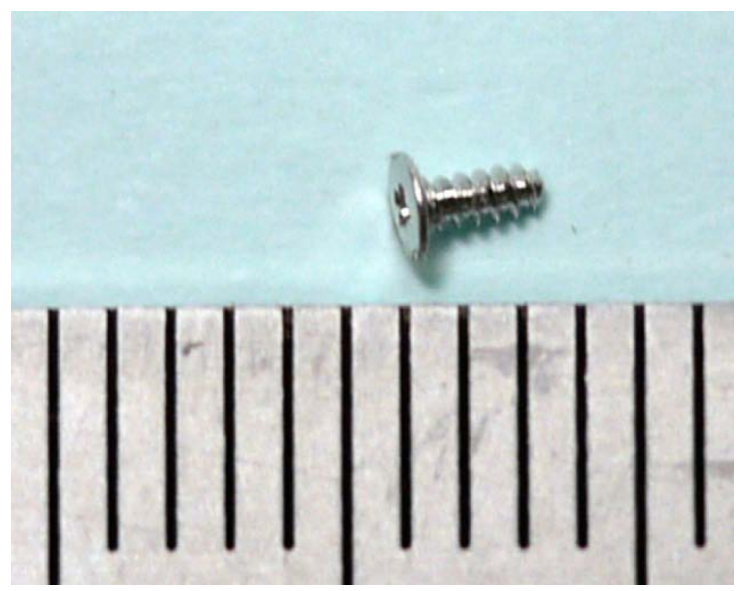

Fig. 2. Platinum-rhodium coated screw $(\varphi 1 \mathrm{~mm}$, length $2.5 \mathrm{~mm}$ ) .

\section{MEASUREMENTS}

The rats were anesthetized with pentobarbital sodium (40mg/kg, ip) again on the following day. The ABRs was measured with the evoked potential measurement system (Synax 1100, NEC). The auditory stimuli were presented with headphones, which were placed closely on the rat's ears.

The conditions of auditory stimuli were listed below.

1. auditory stimulus : click sound

2. stimulus rate $: 9.5 \mathrm{~Hz}$ 
3. stimulus intensity : approximately $100 \mathrm{~dB} \mathrm{spl}{ }^{*}$

4. number of stimulus : 1000times
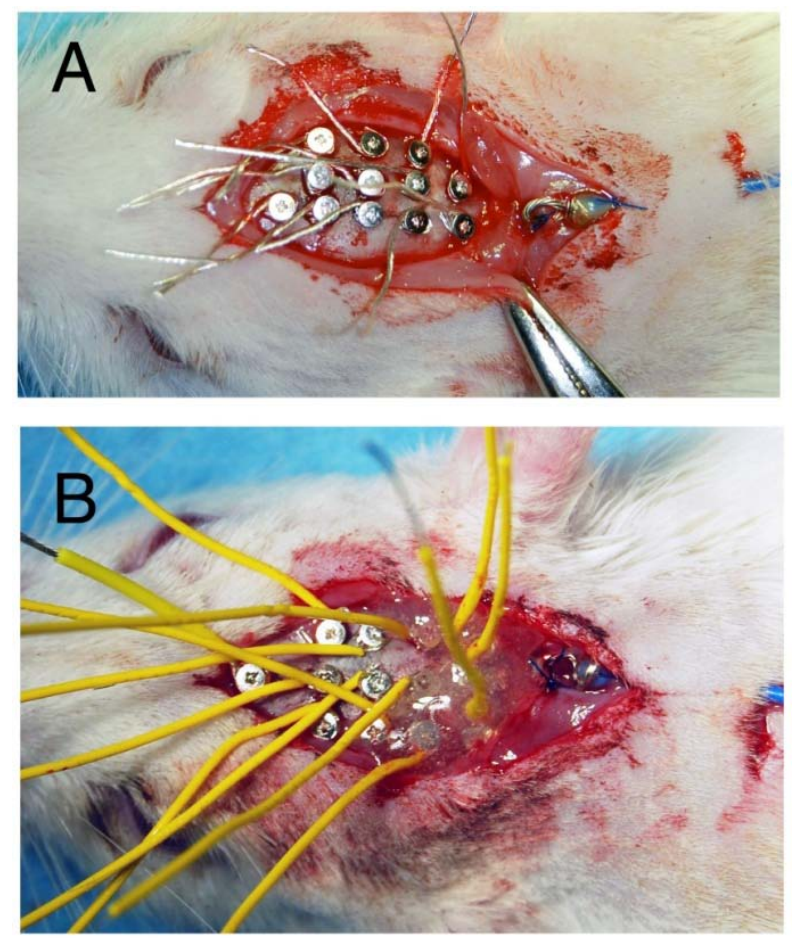

Fig. 3. The photograph of electrodes. In rat B, electrodes were partially covered with epoxy resin.

\section{RESULTS}

Typical ABRs waveform obtained from rat A was shown on Fig. 4. Six positive peaks like humans were observed. Therefore, we named these peaks I to VI according to those of humans.

All ABRs waveforms observed in rat A and B were shown on Fig. 5 and Fig. 6 respectively. When observing these figures attentively, the following points became clear. Firstly, the second peak was observed clearly in all measurement points in both rats. The origin of second peak is thought to be cochlear nucleus in humans. These peaks will fuse together and will become garbled towards the rostral. This phenomenon is not seen in humans. Secondary the morphological structure of each peak was almost same as humans. Mean latency of $\mathrm{V}$ peak in rat was 5.3ms. Lastly, compared with I, II and III, that is, rostral waveforms of ABRs, IV, V and VI might come from caudal part of the brain, which means

* sound pressure level interbrain or brainstem.

From these results, to discriminate these 6 peaks of ABRs waveform, the electrodes on the occipital area were the best.

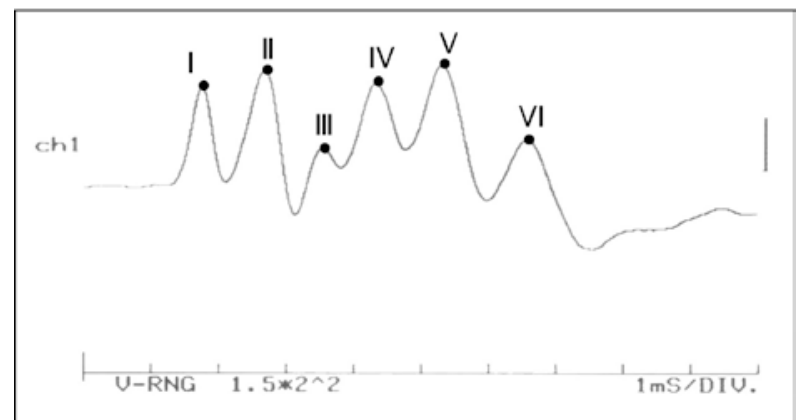

Fig. 4. Typical ABRs waveform measured in rat A. The waveform and morphological structure of each component were similar to that from humans.

\section{DISCUSSION}

Superficial area of 8-week-old rat's skull is so small to implant many electrodes, therefore bare wires were used in rat A. However the distances between these leads were too narrow to connect the connecter of the measurement system. These bare leads sometimes touched to another leads and made noise. In rat $\mathrm{B}$, the operation was complicated compared to bare wire, but teflon coated wires made measurements easier.

We used epoxy resin to fix these electrodes instead of dental cement as many reseachers use. In this model, stable data were obtaind in repeated measurenents over a month. However, we plan to use dental cement in next studies expecting more longer time measurement because some exudate was observed between bone and epoxy resin at sacrifice.

The ABRs were cleary recorded in all electrodes in both rats. In humans, reference electrodes are placed on both side of the ear. In our preliminary study, the reference electrodes were placed on almost same site according to the manner of human ABRs recording. But, obtained resuts were opposite in polarity due to the difference of brain anatomy.

Thus we placed refference electrodes on the latissimus dorsi muscle ${ }^{4-5}$. 


\section{$A B R$ waveforms of rat $A$}
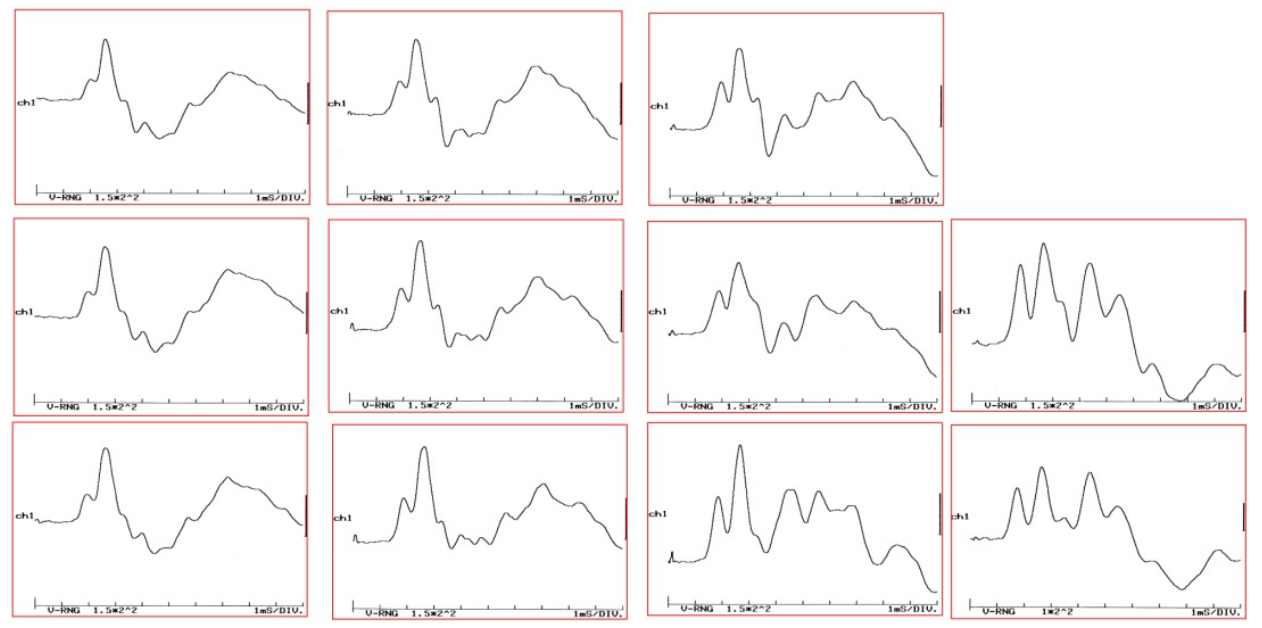

$\begin{array}{lrl}1 & \text { CAL. } \\ 2 & 2 \mu \mathrm{U} \\ 2 & \\ 3 & --- \\ 4 & & ---\end{array}$

$\therefore::$. The figures of each waveform shown above are corresponding to electrode positions

Alignment of electrodes

Fig. 5. All ABRs waveforms recorded in rat A.

\section{$A B R$ waveforms of rat $B$}
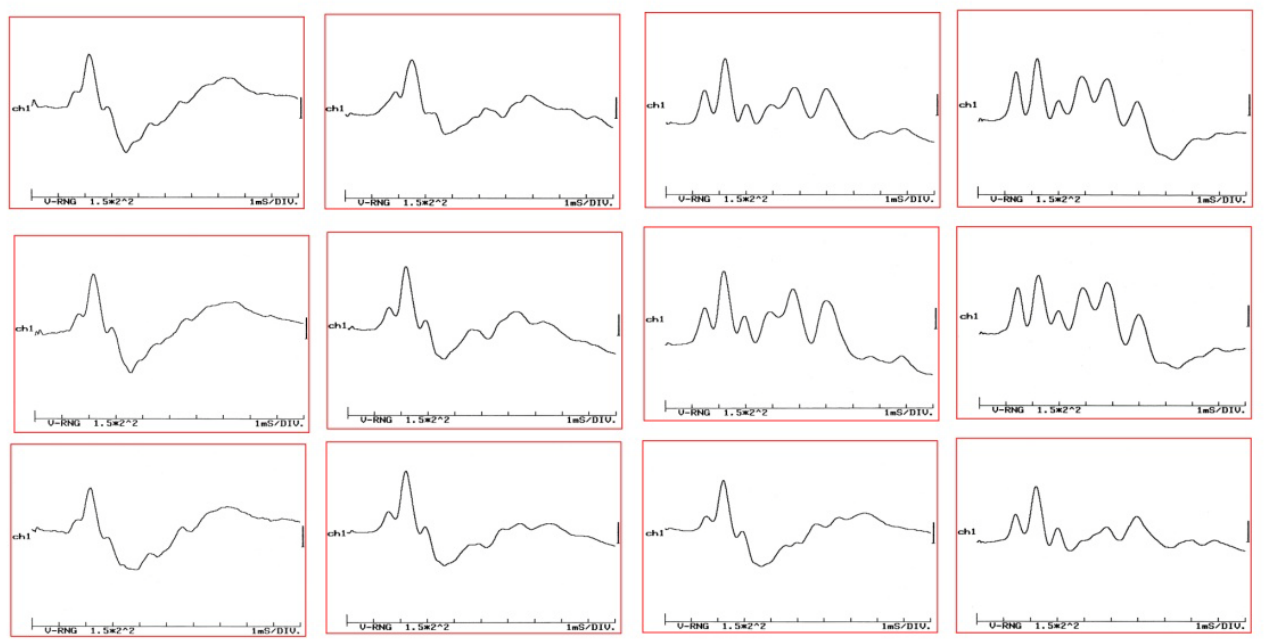

$I^{\infty}$

The figures of each waveform shown above are corresponding to electrode positions

Alignmet of electrodes

Fig. 6. All ABRs waveforms measured in rat B. 
In humans, the measurement electrodes were placed on the $\mathrm{Cz}$ or $\mathrm{Pz}$ (10-20 electrode system), which are center of cranial bone, because the highest voltage of ABRs signal was obtained. However these electrical signal is passed through cranial bone and skin, which are strong barriers. Therefore these potentials passed through bone and skin are named "far field potentials" and do not show localization. However, shown on figures 5 and 6, waveforms of rat's ABRs show localization clearly. Because we used dural electrodes in this paper, these probes detect the celebral electrical activities near the source directly without barriers.

The latency of the each peaks in rats was slightly shorter, compared to humans despite huge difference of body size. This fact suggested that these latencies be mainly due to the numbers of the synapse not length of the axon. According to these, the origin of these peaks may be the same as those of the human's. However, further reserches are needed to determine the source of these ABRs peaks in rat.

In this study, only ABRs was measured. However middle latency responses (MLR) and activities in the auditory cortex were also recorded with dural electrodes as a pilot study. We also have a plan to observe the brain activities by the visual stimulation.

\section{ACKNOWLEDGEMENTS}

We would like to thank Koji Yamada, a teacher of Ichikawa High School, who gave a chance to join this experiment as the project of "Super Science High School” of Ministry of Education, Culture, Sports, Science \& Technology.

\section{REFERENCES}

${ }^{1}$ G. Paxinos(ed.) The Rat Nervous System, $3^{\text {rd }}$ Edition, (Academic Press,ISBN978-0-12-547638-6, 2004) pp.761-1193.

${ }^{2}$ E. Borg and A. Viberg, Age-related hair cell loss in spontaneously hypertensive and normotensive rats, Hearing Res, 30, (1987), pp.111-118.

${ }^{3}$ D.I. Smith, F.S. Lee and J.H. Mills, Middle latency response:Frequency and intesity effects, Hearing Res. 42, (1989), pp. 293-303.

${ }^{4}$ T. Kimura, Y. Serizawa, Y. Hayasaka et al, Latency prolongation of ABRs as recovery from anestheia in rats, in the proceedings of the $36^{\text {th }}$ Congress of the International Union of Physiological Sciences. P3PM-12-3, (2009), p.181.

${ }^{5}$ M. Otsuka, K. Yamazaki, K. Okamoto, et al., Development of ABRs measurement system using chronic implated electrodes in rats (Japanese), No.34 in the proceedings of JSMBE. (2009), p.45.

${ }^{6}$ G. Paxinos and C. Watson The Rat Brain in sterotaxic coordinates, $6^{\text {th }}$ Edittion, (Academic Press ISBN 978-0-12-374243-8, 2009), p.14. 